\title{
GESTIÓN DE RIESGOS EN ESCUELAS PÚBLICAS, CASO PRIMARIA CONSTITUYENTES DE 1857 A 1917
}

\section{RISK MANAGEMENT IN PUBLIC SCHOOLS, ELEMENTARY SCHOOL CONSTITUYENTES CASE FROM 1857 TO 1917}

Recibido: 24 septiembre 2021

Aceptado: 6 noviembre 2021
F. Orduña Correa. ${ }^{1}$

M. C. Rosas Rivera. ${ }^{2}$

J. R. Hernández Atilano. ${ }^{3}$ N.S. Martínez Castillo ${ }^{4}$

\section{RESUMEN}

Toda actividad antrópica implica peligros, por lo cual siempre es necesario antes de emprender una actividad, considerar los riesgos potenciales a los que los individuos deberán enfrentarse al realizar sus actividades, pudiendo así establecer las acciones pertinentes que tiendan a minimizar la ocurrencia y las consecuencias de los mismos. Un sector que es particularmente vulnerable es el infantil, aunque no es un sector que se le permita realizar labores productivas, se encuentran expuestos cuando acuden a las instituciones educativas a realizar sus estudios. Al ser menores de edad, los riesgos pueden incrementarse tanto en frecuencia como en la gravedad de sus consecuencias, siendo indispensable que las instituciones educativas realicen un análisis que permita visualizar todos los factores y así establecer estrategias para proteger tanto a los infantes, como a los docentes y al personal administrativo que labora en las escuelas.

En esta investigación se realizó una evaluacion de riesgos en la escuela primaria "Constituyentes de 1857 a 1917" con el objetivo de establecer una prueba piloto para determinar que tan delicada podría ser la situacion de los individuos dentro de las instalaciones educativas y que acciones podrían ser las pertinentes a contemplar en un plan estratégico que coadyuve a mitigar los riesgos y sus efectos.

PALABRAS CLAVE: Evaluación de Niveles de Riesgo en Escuelas, Consecuencias de Riesgos en Escuelas.

\section{ABSTRACT}

All anthropic activity involves dangers, so it is always necessary before undertaking an activity to consider the potential risks that they will have to face when carrying out their activities, thus being able to establish the pertinent actions that tend to minimize their occurrence and consequences. A sector that is particularly vulnerable is children, although it is not a sector that is allowed to enhance productive work, they are exposed when they go to educational institutions to study. Being minors, the risks can increase both in frequency and in the severity of their consequences, being essential that educational institutions carry out an analysis that allows visualizing all the factors and thus establish strategies to protect both infants and children. teachers and administrative staff working in schools.In this research, a risk assessment was carried out in the elementary school "Constituents from 1857 to 1917" with the aim of establishing a pilot test to determine how delicate the situation of individuals could be within educational facilities and what actions could be those pertinent to contemplate in a strategic plan that helps mitigate risks and their effects.

KEY WORDS: Evaluation of Risk Levels in Schools, Consequences of Risks in Schools.

\footnotetext{
1 Profesor de tiempo completo del Tecnológico Nacional de México, Campus Ciudad Valles, Jefe de Proyectos de Investigación de Ingeniería Industrial. francisco.orduna@tecvalles.mx

2 Profesor de tiempo completo del Tecnológico Nacional de México, Campus Ciudad Valles, carmen.rosas@tecvalles.mx

3 Director de la escuela primaria Constituyentes de 1857 a 1917 joserubenhernandez@upn242.com

4 Estudiante del Tecnológico Nacional de México, Campus Ciudad Valles, 15690508@tecvalles.mx
} 


\section{INTRODUCCIÓN}

Desde comienzos de la humanidad, la actividad antrópica siempre ha implicado riesgos, que han provocado heridas, golpes, amputaciones e incluso la muerte para muchos seres humanos. Con la revolución Industrial se acrecentó el problema involucrando a hombres, mujeres y niños, la recurrencia atrajo la atención de historiadores y de autoridades para desarrollar leyes que permitieran proteger a los trabajadores, sin embargo, mucho antes de la revolución industrial encontramos en el Código de Hammurabi en 1728 el primer indicio legal sobre accidentes de trabajo en el área de la construcción (World History Encyclopedia, 2021). En España, por ejemplo, en 1873 aparece la Ley Benot que prohibía el trabajo en niños menores de 10 años y que limitaba las jornadas de trabajo para los niños entre 10 y 13 años a solo cinco horas. Para 1871 ya aparece un registro que muestra que el $32.1 \%$ de los trabajadores eran niños y el $20.4 \%$ eran niñas, lo que motivó a Eduardo Benot Rodríguez a publicar la Ley Benot, que dentro de los muchos aspectos abarcaba la Protección a la Infancia, La Educación de Menores, Creación de Juzgados Mixtos y la obligatoriedad para las empresas para la contratación de cirujanos, dependiendo del tamaño de la empresa y la cantidad de trabajadores (UNION SINDICAL OBRERA, 2020).

En México la Seguridad y Salud Laboral, se encuentra bajo la protección de la Constitución Política de los Estados Unidos Mexicanos en su artículo 123, y de ahí emanan: Ley De Prevención y Seguridad Escolar del Estado y Municipios de San Luis Potosí (CONGRESO DEL ESTADO DE SLP, 2020) la Ley Orgánica de la Administración Pública Federal, la Ley Federal del Trabajo en su artículo 132, 134 y 512, la Ley Federal sobre Metrología y Normalización en sus artículos 38, 40, 43 al 47, el Reglamento Federal de Seguridad y Salud en el Trabajo y también las Normas Oficiales Mexicanas relativas y otros ordenamientos. Todas estas disposiciones han sido emitidas con el propósito de establecer disposiciones en materia de Seguridad y Salud en el trabajo que eviten riesgos que pongan en peligro la vida, la integridad física o la salud de los trabajadores, así como cambios adversos y sustanciales en el ambiente laboral, que afecten o puedan afectar la seguridad o salud de los trabajadores o provocar daños a las instalaciones, maquinaria, equipos y materiales del centro de trabajo. (Gardner, H. 1973)

Además, la Secretaría de Trabajo y previsión Social (STPS), emite las normas que establecen las condiciones mínimas necesarias para la prevención de riesgos laborales, en atención a los factores a los que puede estar expuesto un trabajador. Por ello la STPS ha emitido normatividades que están encaminadas a observar las diversas condiciones de riesgo ya que cada centro laboral tiene sus propias particularidades, debido a que la naturaleza de la operación puede ser muy diversa en cada caso, actualmente la STPS tiene vigentes 41 normas relativas a los temas de salud y de seguridad e higiene, derivado de la diversidad de operaciones de cada centro laboral, y en consecuencia, es importante observar, que no necesariamente aplican todas ellas en cada caso.

Particularmente en los centros educativos de educación primaria, se observa que existen factores adicionales que no se contemplan en los centros de trabajo tradicionales, que es la presencia de menores de edad, que aun no siendo trabajadores son susceptibles a sufrir riesgos y de los cuales están poco conscientes en cuanto a la prevención, ya que el desenvolvimiento propio de su edad es espontaneo, por lo que no miden el peligro, exponiéndose a sufrir riesgos que pueden ser desde pequeñas caídas con rapaduras leves hasta 
golpes graves e incluso mortales, por lo que se requiere una atención especial para identificar analizar y prevenir los riesgos y los factores de riesgo que hacen vulnerable la seguridad de la niñez mexicana y de los adultos que están a cargo de ellos (SEP, 2018). La NOM-009SEGOB-2015 define los riesgos "como daños o pérdidas probables sobre un agente afectable, resultado de la interacción entre su vulnerabilidad y la presencia de un agente perturbador" (SEGOB, 2018). Para el gobierno de México es fundamental garantizar la integridad física y emocional de los niños, las niñas y los adolescentes por lo que ha emitido guías para la prevención de riesgos de modo que se adopten medidas prácticas que involucren incluso a los padres de familia con el objetivo de mitigar hasta donde sea posible los efectos que pudieran provocar los riesgos a los que están expuestos los estudiante (CENAPRED/INIFED, 2018), ya que es en la escuela donde pasan gran parte de su vida infantil. Por otra parte, es destacable el hecho de que México es un país muy diverso y los niveles de exposición al riesgo son muy variables dependiendo de cada región, sin embargo, aun en los lugares más remotos existen ocurrencias de riesgos y se requiere estar preparados para prevenir, mitigar y responder a las emergencias que se pudieran presentar (SEMARNAT, 2013).

Con el surgimiento de la contingencia sanitaria provocada por el COVID 19 (SARS COV II), y la incertidumbre de los contagios, las acciones para prevenir los riesgos y disminuir sus efectos se hicieron prioritarias, haciendo de esta intervención vinculatoria una gestión oportuna que fue muy bien recibida tanto por las autoridades de la Unidad Regional de Servicios Educativos Huasteca Norte (URSE) como por el responsable de la institución y todos sus colaboradores.

En esta investigación se aborda el caso de una escuela primaria ubicada en Ciudad Valles S.L.P. cuya trayectoria académica ha sido trascendental en la región, en sus aulas se han formado generaciones de niños y niñas que no han estado exentos a contingencias de riesgo, y que afortunadamente no han dejado consecuencias graves sin embargo, con el trascurso del tiempo la infraestructura física de las instalaciones se ha demeritado por el uso y el pasar de los años, observando áreas de oportunidad importantes que deben atenderse para garantizar la seguridad e integridad física de toda su población.

La escuela primaria “Constituyentes de 1857 a 1917". fue fundada en las instalaciones del actual mercado Constitución en el año de 1959, fungiendo como directora la maestra Eusebia Ramos Moctezuma, quien en compañía de otras profesoras dieron apertura a las primeras clases con pocos alumnos divididos en cuatro salones; con el pasar del tiempo fue insuficiente el espacio llegando a impartir clases en el patio, inclusive se cuenta que salían hasta el malecón del río, lo que le daba un toque de esparcimiento y contacto con la naturaleza a las enseñanzas. Sin embargo, la ubicación en el área de los mercados no fue las mas adecuada ya que por esos años, esos espacios eran considerados los de mayor movimiento de la población, además del crecimiento de las actividades comerciales en torno a la escuela la hacían crear un ambiente de incomodidad entre otras cosas por la contaminación que propiciaba la basura arrojada por la dinámica del comercio. En 1960 la escuela pasa del orden municipal al estatal y posteriormente al federal. En 1963 con la obra altruista de la señora Josefina Morales Corzo quien dona el terreno y con las gestiones del C. Reynaldo Lara Trejo logran cambiar su ubicación a su actual sede, en la colonia Vergel en donde a base de esfuerzo, especialmente en los primeros años donde las condiciones eran complejas, terreno enmontado y anidado de serpientes, logran continuar con la noble labor de impartir clases y seguir formando seres humanos de provecho. Es hasta mediados de los años sesenta 
que el alcalde Mario Loustau Álvarez gestiona la construcción del edificio inicial que fue la base de lo que hoy existe de infraestructura. (Panorama 2009). Actualmente la institución con 62 años es una de las escuelas primarias más dinámicas y propositivas de la región, abierta a las propuestas de mejora continua y con un liderazgo transformacional que se hace evidente en su dinámica escolar. Cuenta con una población 360 alumnos de primero a sexto grado en el turno matutino, equipos de basquetbol y futbol, 17 maestros frente a grupo, dos profesores de educación física, una maestra de apoyo, una psicóloga, dos asistentes de mantenimiento y un director.

\section{METODOLOGÍA}

Se lleva a cabo la investigación tecnológica aplicada a través de la metodología de gestión de riesgos laborales, la cual nos indica que es necesario primeramente realizar una análisis que involucre la política preventiva de la institución, la organización preventiva, la planificación y la revisión de actuaciones, establecidos como un Plan de Prevención; estos aspectos quedan cubiertos dentro de los objetivos y elementos clave del Programa Nacional de Convivencia Escolar (SEGOB, 2019) que está destinado a regular todos los aspectos relacionados con la convivencia de estudiantes, docentes y administrativos y con la estrategia nacional para el regreso seguro a las escuelas de educación básica (SEP 2021).

Se formaliza la intervención por parte del Tecnológico Nacional de México campus Ciudad Valles con la firma de los convenios de colaboración correspondientes y las facilidades de las autoridades académicas de la escuela primaria Constituyentes de 1857 a 1917. Se llevan a cabo las visitas de campo para conocer las áreas e identificarlas para el posterior diseño del layout que facilite la definición de espacios. Se realizan reuniones de retroalimentación con el director del plantel para conocer los antecedentes de la infraestructura física relacionados con la antigüedad y antecedentes de su construcción, la historia de sus mantenimientos, los motivos de su deterioro y las posibilidades de reparación. Se indaga de igual manera la dinámica de actividades realizadas diariamente tanto por los estudiantes como por el personal docente.

Se revisa el marco legal de normatividades y reglamentos relacionados con los riesgos escolares, así como los fundamentos legales relacionados con la gestión integral de riesgos para los centros educativos.

Se lleva a cabo el diagnostico de vulnerabilidad y evaluación de riesgos. Los parámetros para determinar el nivel de riesgo, así como la probabilidad de que algo negativo ocurra se calcula con esta metodología considerando lo siguiente:

Tabla 1. Parámetros para determinar el nivel de riesgo y probabilidad de que ocurra Fuente: SSER 2013

1) El nivel de probabilidad de que materialice el riesgo

2) El nivel de consecuencias o impacto esperado si se materializa el riesgo

3) El nivel de deficiencias encontradas

4) El nivel de exposición de las personas al riesgo 
Para establecer los niveles de riesgo se utilizaron distintas listas de chequeo donde se analizaron los factores de riesgo para cada situación, en donde se obtienen tres posibles respuestas: a) S si cumple b) N no cumple c) X no aplica

Una vez llenados los cuestionarios se realiza la estimación del nivel de deficiencias (ND) con la siguiente formula:

Porcentaje de deficiencias $=$ \# de criterios que no cumplen*100/núm. Total de criterios que Aplican.

El Total de deficiencias que aplican es la diferencia del total de criterios, por cada riesgo, menos el número de criterios calificados como NO aplican. Definido el porcentaje de deficiencias, se revisa la tabla siguiente y se estima el Nivel de deficiencias:

Tabla 2. Índices de desempeño del nivel de deficiencias ND para los riesgos asociados a las actividades escolares. Fuente: DGSSL 2004

\begin{tabular}{|c|c|c|}
\hline $\begin{array}{l}\text { Porcentaje de deficiencias } \\
\qquad \%\end{array}$ & Estimación del ND & Significado \\
\hline $70<\% \leq 100$ & $\begin{array}{l}\text { Muy deficiente } \\
\text { MD }\end{array}$ & $\begin{array}{l}\text { Se ha detectado que el } 71 \% \text { o más de deficiencias y factores } \\
\text { de riesgo deben ser corregidos, ya que determinan como } \\
\text { muy posible la generación de accidentes. El conjunto de } \\
\text { medidas preventivas existentes respecto al riesgo resulta } \\
\text { ineficaz. }\end{array}$ \\
\hline $40<\% \leq 70$ & $\begin{array}{l}\text { Deficiente } \\
\text { D }\end{array}$ & $\begin{array}{l}\text { Se ha detectado que entre el } 31 \% \text { y el } 70 \% \text { de deficiencias } \\
\text { y factores de riesgo precisan ser corregidos. La eficiencia } \\
\text { del conjunto de medidas preventivas existentes se ve } \\
\text { reducida de forma apreciable. }\end{array}$ \\
\hline $0<\% \leq 40$ & $\begin{array}{l}\text { Mejorable } \\
\mathrm{M}\end{array}$ & $\begin{array}{l}\text { Se ha detectado que hasta el } 30 \% \text { de deficiencias y factores } \\
\text { de riesgo deben ser corregidos. La eficiencia del conjunto } \\
\text { de medidas preventivas existentes respecto al riesgo no se } \\
\text { ve reducida de forma apreciable. }\end{array}$ \\
\hline $0<\% \leq 10$ & $\begin{array}{c}\text { Aceptable } \\
\text { A }\end{array}$ & $\begin{array}{l}\text { No se ha detectado un número de deficiencias ni factores } \\
\text { de riesgo relevantes. El riesgo está controlado. }\end{array}$ \\
\hline
\end{tabular}

Después de realizar la evaluación de los riesgos se elabora el Mapa de riesgos con la señalización correspondiente y se elabora una propuesta para corregir los hallazgos observados, así como la elaboración de un presupuesto estimado que permitirá realizar una planificación y gestión de recursos para esos fines.

\section{RESULTADOS}

La escuela primaria Constituyentes de 1857 a 1917 cuenta con cinco edificios, en los cuales se ubican 18 salones para la impartición de clases, 03 módulos para sanitarios, una oficina administrativa donde se encuentra la dirección, una plaza cívica techada en donde se llevan a cabo actividades deportivas y culturales, una explanada con mesas de piedra en los 
alrededores, una cancha de basquetbol a cielo abierto, un campo deportivo, 03 bodegas de almacenamiento y un estacionamiento. No se cuenta con un registro de accidentes, pero se tiene conocimiento de que han ocurrido. En las indagatorias con el personal de mayor antigüedad en la institución comentan que las consecuencias han sido desde raspones por caídas hasta perdida de extremidades derivado de los factores de riesgo que provocan los desniveles del piso. Dentro de las instalaciones se encuentran arboles frutales los cuáles además de generar desechos orgánicos representan un alto riesgo provocando caídas y resbalones.

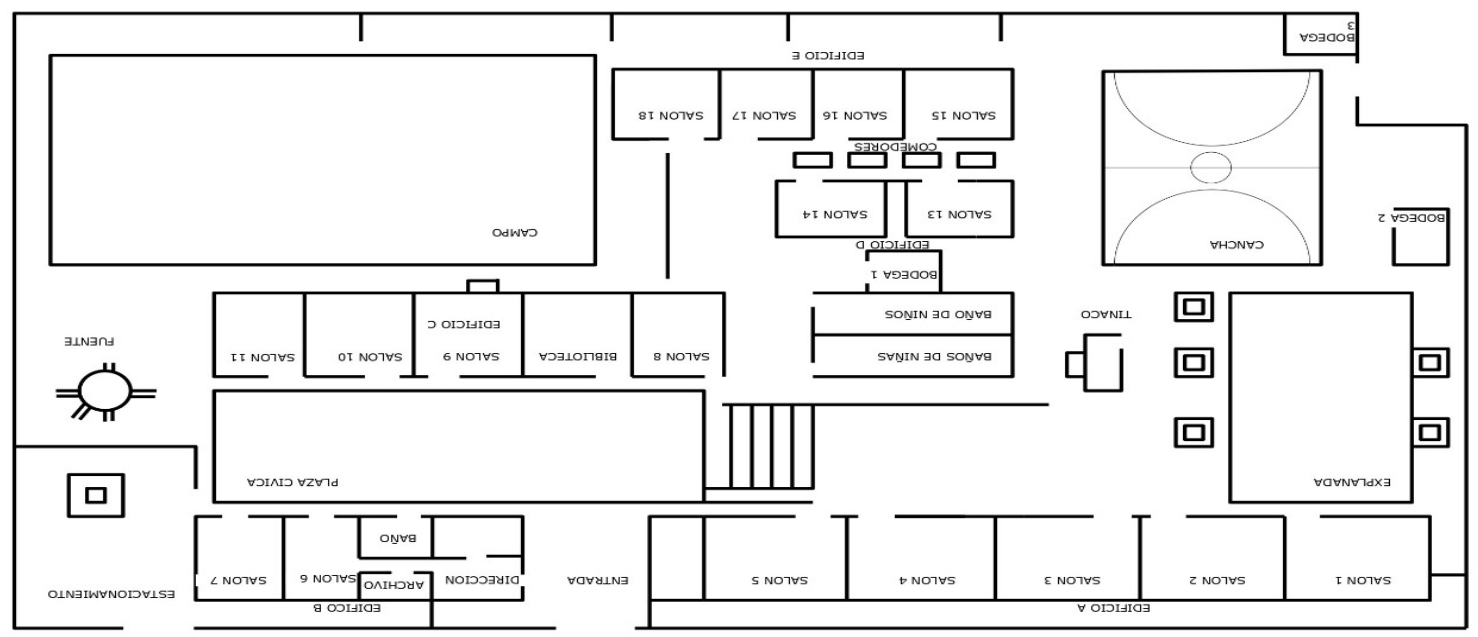

Figura 1. Plano de la Escuela primaria Constituyentes de 1857 a 1917 Fuente: Elaboración propia

Se identifican riesgos que han sido protagonizados por los estudiantes, personal docente y administrativo como caídas al mismo nivel provocadas por tropezar o resbalar, caídas a desnivel, choques o golpes contra objetos inmóviles como es el caso de las mesas de piedra que fungen como bancas de esparcimiento, o en los objetos que se encuentran en los pasillos y con los cuales los niños se topan cuando van corriendo, contactos eléctricos en mal estado, accidentes provocados en los desplazamientos prolongados a las aulas, falta de iluminación en las aulas, ruido excesivo proveniente de las aulas cercanas o de la calle, vibraciones por la existencia de obras cercanas, trastornos musculo-esqueléticos en el caso de los profesores por permanecer mucho tiempo en una misma posición.

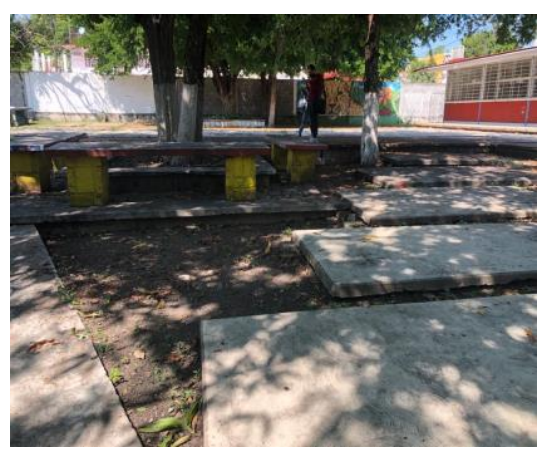

Figura 2. Desniveles mal planeados en los patios. Fuente: Propia

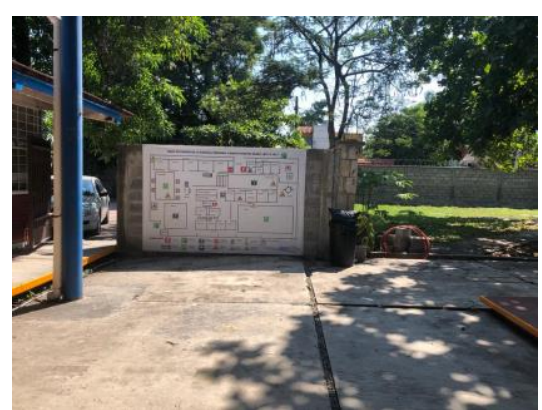

Figura 3. Instalación de mapa de riesgos de la institución. Fuente: Propia 
Después de hacer el diagnostico de vulnerabilidad y la evaluación de riesgos se identifican nueve riesgos con una probabilidad de ocurrencia muy alta. Con base a este criterio y a la determinación de riesgos en la Escuela Primaria Constituyentes de 1857 a 1917 se encontró lo siguiente:

Tabla 3. Resultado del Nivel de deficiencias ND, para los riesgos asociados a las actividades escolares. Fuente: Elaboración Propia

\begin{tabular}{|c|c|c|c|c|c|}
\hline NO & RIESGOS & $\begin{array}{l}\text { ACEPTABLE } \\
\text { A }\end{array}$ & $\begin{array}{l}\text { MEJORABLE } \\
\text { M }\end{array}$ & $\begin{array}{l}\text { DEFICIENTE } \\
\text { D }\end{array}$ & $\begin{array}{l}\text { MUY } \\
\text { DEFICIENTE } \\
\text { MD }\end{array}$ \\
\hline 1 & CAÍDAS & & & $X=62.5 \%$ & \\
\hline 2 & $\begin{array}{c}\text { GOLPES, CORTES Y } \\
\text { HERIDAS }\end{array}$ & & & $X=64.28 \%$ & \\
\hline 3 & ELECTROCUCIÓN & & $\mathrm{X}=11.11$ & & \\
\hline 4 & $\begin{array}{l}\text { ENVENENAMIENTO, } \\
\text { ELECTROCUCIÓN }\end{array}$ & $X=0 \%$ & & & \\
\hline 5 & $\begin{array}{l}\text { INCENDIOS Y } \\
\text { EXPLOSIONES }\end{array}$ & & & $X=50 \%$ & \\
\hline 6 & $\begin{array}{l}\text { ACCIDENTES DE } \\
\text { TRÁNSITO }\end{array}$ & & & $X=62.5 \%$ & \\
\hline 7 & $\begin{array}{c}\text { ENFRENTAMIENTO CON } \\
\text { ARMAS DE FUEGO EN LAS } \\
\text { INMEDIACIONES DE LA } \\
\text { ESCUELA }\end{array}$ & & & $X=42.5 \%$ & \\
\hline 8 & $\begin{array}{c}\text { PRESENCIA DE ARMAS EN } \\
\text { LA ESCUELA }\end{array}$ & & $X=37.5 \%$ & & \\
\hline 9 & AMENAZAS Y EXTORSIÓN & & $\mathrm{X}=33.33 \%$ & & \\
\hline 10 & $\begin{array}{c}\text { PRESENCIA Y CONSUMO } \\
\text { DE DROGAS EN EL } \\
\text { ENTORNO }\end{array}$ & & $X=33.33 \%$ & & \\
\hline 11 & ROBO Y HURTO & & $X=25 \%$ & & \\
\hline 12 & SECUESTRO & & $\mathrm{X}=12.5 \%$ & & \\
\hline
\end{tabular}

En base a esta información y realizando el cruce con el análisis de la probabilidad de que ocurra un evento, y comparado con el nivel de consecuencias, se obtuvo la siguiente tabla que permite visualizar en forma completa la situación actual de la institución.

Tabla 4. Nivel de Riesgo identificado en la escuela Constituyentes de 1857 a 1917. Fuente: Propia

\begin{tabular}{|c|c|c|c|c|c|c|c|}
\hline NO & RIESGOS & $\begin{array}{c}\text { NIVEL DE } \\
\text { PROBABILIDAD } \\
\text { NP }\end{array}$ & $\begin{array}{c}\text { NIVEL DE } \\
\text { CONSECUENCIAS } \\
\text { NC }\end{array}$ & $\begin{array}{l}\text { RIESGO } \\
\text { BAJO }\end{array}$ & $\begin{array}{l}\text { RIESGO } \\
\text { MEDIO }\end{array}$ & $\begin{array}{c}\text { RIESGO } \\
\text { ALTO }\end{array}$ & $\begin{array}{c}\text { RIESGO } \\
\text { MUY } \\
\text { ALTO }\end{array}$ \\
\hline 1 & CAÍDAS & PA & MG & & & & $\mathrm{X}$ \\
\hline 2 & $\begin{array}{c}\text { GOLPES, CORTES Y } \\
\text { HERIDAS }\end{array}$ & PA & MG & & & & $\mathrm{X}$ \\
\hline 3 & ELECTROCUCIÓN & PM & MG & & & $\mathrm{X}$ & \\
\hline 4 & $\begin{array}{l}\text { ENVENENAMIENTO } \\
\text { ELECTROCUCIÓN }\end{array}$ & PB & M & & & $\mathrm{X}$ & \\
\hline
\end{tabular}




\begin{tabular}{|c|c|c|c|c|c|c|c|}
\hline NO & RIESGOS & $\begin{array}{c}\text { NIVEL DE } \\
\text { PROBABILIDAD } \\
\text { NP }\end{array}$ & $\begin{array}{c}\text { NIVEL DE } \\
\text { CONSECUENCIAS } \\
\text { NC }\end{array}$ & $\begin{array}{c}\text { RIESGO } \\
\text { BAJO }\end{array}$ & $\begin{array}{l}\text { RIESGO } \\
\text { MEDIO }\end{array}$ & $\begin{array}{l}\text { RIESGO } \\
\text { ALTO }\end{array}$ & $\begin{array}{l}\text { RIESGO } \\
\text { MUY } \\
\text { ALTO }\end{array}$ \\
\hline 5 & $\begin{array}{l}\text { INCENDIOS Y } \\
\text { EXPLOSIONES }\end{array}$ & PA & M & & & & $\mathrm{X}$ \\
\hline 6 & $\begin{array}{l}\text { ACCIDENTES DE } \\
\text { TRÁNSITO }\end{array}$ & PA & M & & & & $\mathrm{X}$ \\
\hline 7 & $\begin{array}{l}\text { ENFRENTAMIENTO } \\
\text { CON ARMAS DE } \\
\text { FUEGO EN LAS } \\
\text { INMEDIACIONES } \\
\text { DE LA ESCUELA }\end{array}$ & PMA & M & & & & $\mathrm{X}$ \\
\hline 8 & $\begin{array}{l}\text { PRESENCIA DE } \\
\text { ARMAS EN LA } \\
\text { ESCUELA }\end{array}$ & PM & M & & & & $\mathrm{X}$ \\
\hline 9 & $\begin{array}{l}\text { AMENAZAS Y } \\
\text { EXTORSIÓN }\end{array}$ & PM & MG & & & $\mathrm{X}$ & \\
\hline 10 & $\begin{array}{c}\text { PRESENCIA Y } \\
\text { CONSUMO DE } \\
\text { DROGAS EN EL } \\
\text { ENTORNO }\end{array}$ & PM & M & & & & $\mathrm{X}$ \\
\hline 11 & ROBO Y HURTO & PM & M & & & & $\mathrm{X}$ \\
\hline 12 & SECUESTRO & PM & $\mathrm{M}$ & & & & $\mathrm{X}$ \\
\hline
\end{tabular}

Con estos datos y con la designación de claves para identificar los espacios, se elaboró el mapa de riesgos, el cual se imprimió en una lona grande que fue patrocinada como una donación por parte del Club Rotario Tantocob en contribución a la educación pública colocándose en la plaza cívica de la institución, fortaleciendo con ello los procesos vinculatorios interinstitucionales.

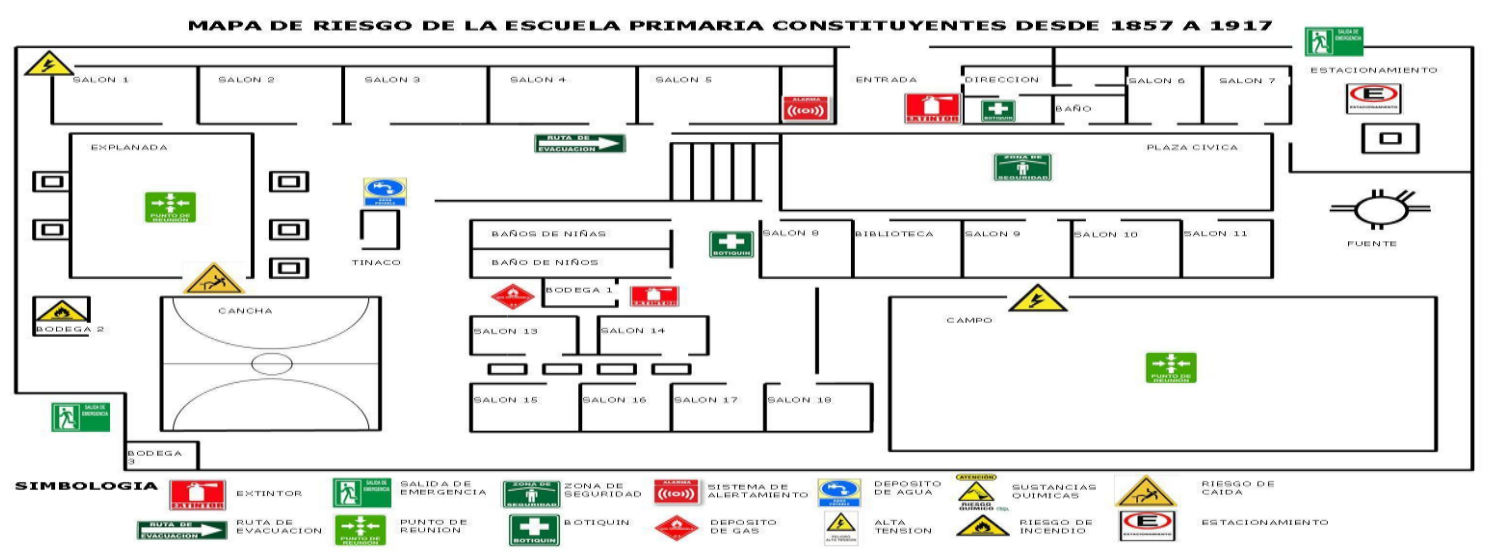

Figura 4. Mapa de Riesgos de la Escuela Constituyentes de 1857 a 1917 Fuente: Propia 
Uno de los riesgos identificados más significativos por el nivel de exposición, fue el de la condición de la barda perimetral, la cual tiene una altura muy accesible representando una amenaza para la seguridad tanto de las personas como del inmueble, por lo que uno de los proyectos de mejora de la institución fue la inversión significativa en la instalación de concertina en todo el perímetro de la barda. De igual manera se realizaron acciones de mejora y mantenimiento en las instalaciones como fue la reparación de techos, instalaciones eléctricas, pintura de edificios y áreas de recreación, canchas deportivas, mantenimiento al campo deportivo y señalización de áreas y líneas donde puede haber riesgos, apegados a la NOM-003-SEGOB-2011 (2011), señales y avisos para protección civil colores, formas y símbolos a utilizar.

En relación con la prevención para evitar la propagación del SARS-CoV-2 se siguen los protocolos establecidos por el sector salud para evitar los contagios y con ello garantizar el regreso seguro a las clases y con el menor riesgo posible manteniendo el control en los accesos de la institución, el monitoreo, la sanitización y ventilación adecuados.

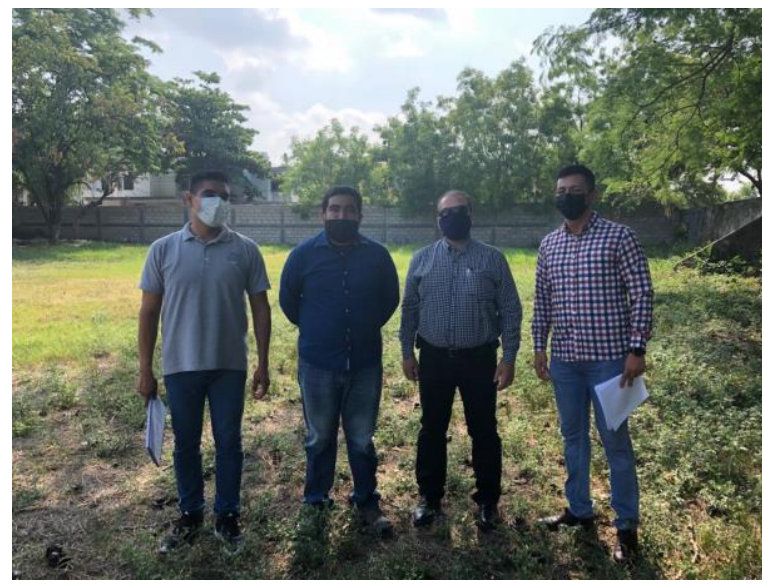

Figura 5. Inspección de Instalaciones e Identificación de riesgos. Fuente Propia

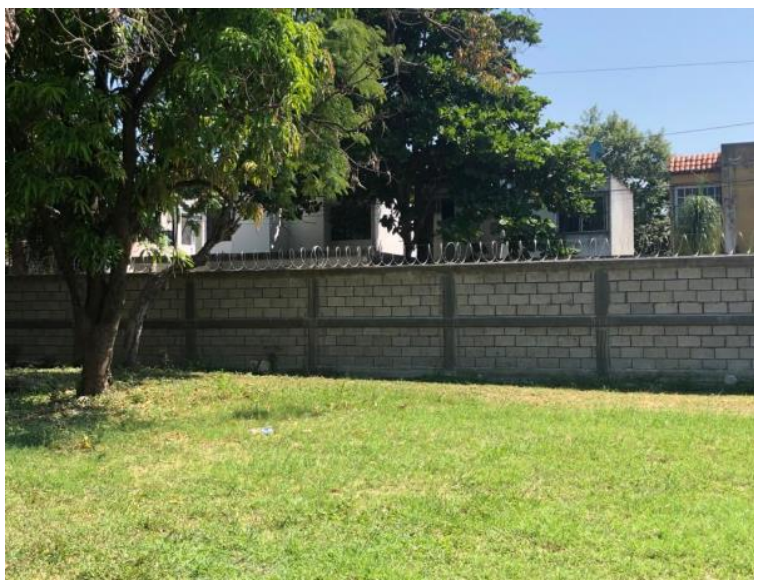

Figura 6. Barda Perimetral con concertina. Fuente: Propia

\section{CONCLUSIONES}

Los riesgos escolares son más comunes de lo que creemos y de lo que estamos conscientes la mayoría de la población educativa, padres de familia, tutores y autoridades escolares. Sin embargo, es un tema relegado que se maneja con una actitud reactiva, se interviene en cuanto surge el problema, pero se adolece de una cultura de prevención y no se cuenta con capacitación adecuada para resolver las emergencias que se pueden presentar ante la ocurrencia de un riesgo. Por otra parte, no es tan accesible lograr implementar la capacitación formal en esos temas ya que en su gran mayoría los profesionales de la seguridad e higiene facultados para preparar recursos humanos con esas competencias requieren del pago de honorarios y en una gran proporción de casos no se tienen destinados recursos para esos fines. Es relevante destacar que estas capacitaciones requirieren de conocimientos sistemáticos basados en técnicas específicas, como es el soporte vital básico, la atención de primeros auxilios, la manipulación y el transporte de heridos entre otros; que pueden hacer la diferencia entre la vida y la muerte. En el desarrollo de la investigación se revisaron los datos relacionados con las instituciones que tienen desarrollados programas de seguridad y salud 
escolar, de acuerdo a la Unidad Regional de Servicios Educativos Huasteca Norte (URSE) se cuenta con un registro de 101 escuelas públicas en el municipio de Ciudad Valles, de las cuáles ninguna tiene implementado un programa de identificación y prevención de riesgos y además desconocen el tema, por lo que la escuela primaria Constituyentes de 1857 a 1917 , es la primera institución pública en contar con un programa de gestión integral de riesgos.

Se considera importante puntualizar que de los riesgos evaluados en el centro educativo nueve fueron catalogados con riesgo muy alto entre los que se encuentran caídas, golpes, heridas, incendios y accidentes de tráfico, como riesgos altos se determinaron electrocución y envenenamiento y como riesgos potenciales los asociados a la violencia social entre los que se encuentran enfrentamientos con armas de fuego en los alrededores de las instalaciones, amenazas y extorsión (SEGOB, 2018). Se recomienda continuar con los esfuerzos para mantener las acciones de mejora ya que se requiere seguir invirtiendo o consiguiendo el patrocinio para contar con un botiquín equipado, extinguidores para combatir conatos de incendio (STPS, 2010)y mantener los programas de mantenimiento preventivo y correctivo a la infraestructura física, especialmente al piso que presenta hundimientos y desniveles y hacer de la seguridad y salud escolar una cultura que forme parte de los aprendizajes claves en su modelo educativo.

\section{BIBLIOGRAFÍA}

CENAPRED/INIFED. (2018). Guía para elaborar o actualizar el programa interno de protección civil escolar. Recuperado el Febrero de 2021, de proteccióncivil.cdmx.gob.mx: https://www.gob.mx/cms/uploads/attachment/file/419325/Tercera_Edici_n_de_la_Gu_a_P C-2018.pdf

CONGRESO DEL ESTADO DE SLP. (2020). Ley De Prevencion Y Seguridad Escolar Del Estado Y Municipios De San Luis Potosi. Obtenido de vlex.com.mx: https://vlex.com.mx/vid/leyprevencion-seguridad-escolar-593753878

Gardner, H. (1973). Las artes y el desarrollo humano. Nueva York.: Wiley.

Mariana Robles, Gustavo Wilches. (s.f.). Gestion del Riesgo en la Escuela. Obtenido de BID: https://publications.iadb.org/en/publications/spanish/document/Gesti\%C3\%B3n-del-riesgoen-la-escuela.pdf

CENAPRED Riesgo, A. N. (25 de 06 de 2021). Monitoreo de Avisos y Fenomenos Naturales. Obtenido de http://www.atlasnacionalderiesgos.gob.mx

SEGOB. (23 de 12 de 2011). NOM-003-SEGOB-2011. Diario Oficial de la Federación. Ciudad de México, Ciudad de México, México: Gobierno Federal.

SEGOB. (2018). Guía para la prevención, detección y reacción ante la presencia de armas enlas escuelas. Recuperado el sep de 2021, de www.gob.mx:

https://www.gob.mx/cms/uploads/attachment/file/342152/Guia_prevencion_de_armas_en_1 as_escuelas.pdf

SEGOB. (5 de Julio de 2018). NOM-009-SEGOB-2015. Diario Oficial de la Federación. Ciudad de México, Ciudad de México, México: GObierno Federal.

SEGOB. (28 de 12 de 2019). Reglas de Operación del Programa Nacional de Convivencia Escolar. Diario Oficial de la Federación. Ciudad de México, Ciudad de México, México: Gobierno Federal. 
SEMARNAT. (01 de 02 de 2013). NOM-161-SEMARNAT-2011. Diario Oficial de la Federación. Ciudad de México, Ciudad de México, México: Gobierno Federal.

SEP. (2018). Guía para elaborar o actualizar el programa escolar de protección civil. Instituto Naional de Infraestructura Física Educativa. Ciudad de México: Gobierno Federal.

STPS. (9 de 12 de 2010). NOM-002-STPS-2010. Diario Oficial de la Federación. Ciudad de México, Ciudad de México, México: Gobierno Federal.

Union Sindical Obrera. (2020). Ley Benot, primera ley del Derecho del Trabajo en España. Recuperado el SEPTIEMBRE de 2021, de uso.es: https://www.uso.es/ley-benot-primeraley-del-derecho-del-trabajo-en-espana/

World History Encyclopedia. (2021). El código de Hammurabi. Recuperado el agosto de 2021, de worldhistori.com: https://www.worldhistory.org/trans/es/1-19882/el-codigo-de-hammurabi/ 